\title{
Minimização de caixa preta pelos métodos de Steepest Descent e Simulated Annealing
}

\author{
Marques, T. A. C. ${ }^{1^{*}}$; Galo, Y.G. ${ }^{1}$; Silva, W. B. ${ }^{2}$ \\ 1 Curso de Graduação em Engenharia Química, Universidade Federal do Espírito Santo, Alegre, ES, Brasil. \\ 2 Programa de Pós-Graduação em Engenharia Química, Curso de Graduação em Engenharia Química, Departamento \\ de EngenhariaRural, Universidade Federal do Espírito Santo, Alegre, ES, Brasil. \\ *e-mail: tacianecolamarques@gmail.com
}

\begin{abstract}
Resumo
A otimização pode ser utilizada para minimizar funções, com o objetivo de obter um processo mais eficiente. Funções que armazenam dados relacionados ao funcionamento de um processo sem revelar o comportamento que a função objetivo apresenta, são denominadas de funções caixa-preta. Para a minimização dessas funções podem ser utilizados métodos como Steepest Descent e Simulated Annealing, sendo o primeiro denominado como um método determinístico, pois ao utilizá-lo, o resultado obtido sempre será o mesmo quando o mesmo ponto inicial for utilizado, e o segundo é uma técnica metaheurística de busca local, originada do processo de recozimento de metais, e que nem sempre retorna o mesmo valor quando utiliza-se o mesmo ponto inicial. Dessa forma o presente trabalho tem como objetivo a minimização da função caixa preta utilizando dois métodos computacionais: Steepest Descent e Simulated Annealing.
\end{abstract}

\begin{abstract}
The optimization can be used to minimize functions in order to obtain/get a more efficient process. Functions that store data related to the functioning of a process without revealing the behavior that the objective function presents, are called blackbox functions. To minimize these functions methods as Steepest Descent and Simulated Annealing may be used. The first one is called a deterministic method because when using it, the result obtained always will be the same when the same starting point is used. The second one is a metaheuristic technique of local search originated from the process of annealing metals, which not always return to the same value when it uses the same starting point. Thus the present work aims to minimizing the blackbox function using two computational methods: Steepest Descent and Simulated Annealing.
\end{abstract}

Keywords (Palavras chaves): minimização, Steepest Descent, Simulated Annealing.

\section{Introdução}

Otimizar é tornar um processo mais eficiente com o menor custo. Nos casos mais simples consiste em minimizar ou maximizar uma função real [1]. A otimização pode ser utilizada em diferentes áreas do conhecimento: negócios, ciências físicas, químicas e biológicas, engenharia, arquitetura, economia e administração. O número de aplicações é tão extenso quanto à quantidade de ferramentas disponível para auxiliar nesse processo de otimização [2].
Com o avanço da tecnologia computacional, inúmeras técnicas de otimização evoluíram e com o desenvolvimento da inteligência computacional, muitas técnicas Heurísticas e Metaheurísticas também tem sido utilizadas para a solução desses problemas. Essas técnicas, além de apresentar rápida convergência possuem também baixo custo computacional, fácil formulação e implementação [1].

A heurística é uma técnica de otimização com etapas bem definidas, onde para problemas complexos são 
encontradas soluções de boa qualidade. Porém, o processo termina quando encontra um ótimo local, pois os vizinhos testados ao redor desse valor adotado são piores. Dessa forma, essas soluções encontradas,

geralmente, não são ótimos globais [3]. O método Metaheurístico, segundo Romero et al. (2004), pode ser considerado como uma evolução dos algoritmos heurísticos. Isso porque o método Metaheurístico possui mecanismos internos que evitam parar em ótimo local, mesmo que para isso piorem a solução objetiva.

A função objetivo é uma função onde cada ponto do espaço de soluções está associado a um número real. Sendo a qualidade da resposta medida por esse número real, ou seja, em um problema de minimização, quanto menor esse valor, melhor a resposta. E em um processo de maximização, o inverso [2].

Quando o conhecimento sobre um processo, e consequentemente sobre a função objetivo que o descreve é restrito, tem-se uma função chamada de caixa-preta. Esta função armazena os dados relacionados ao funcionamento de um processo sem revelar o comportamento que a função objetivo apresenta. Na otimização de processos utilizando uma função caixa-preta, é necessário apenas o conhecimento das entradas desse sistema, que são os parâmetros do processo, e assim é possível obter a resposta, as saídas, que o sistema em estudo apresenta [4].

Para a minimização ou maximização de funções objetivos podemos utilizar diferentes métodos existentes na literatura. Neste trabalho será utilizado dois métodos para a minimização da função objetivo, são eles o método Steepest Descent e o método Simulated Annealing.

O método Steepest Descent, muitas vezes chamado de método Gradient Descent [5], compreende-se dentre os métodos mais antigos e conhecidos e que são usados para a minimização de funções que apresentam várias variáveis [6]. Este método, considerado o mais simples dos métodos do tipo gradiente, foi criado em 1847 por Cauchy [5], e é denominado como um método determinístico, ou seja, utilizando-o, o resultado obtido sempre será o mesmo quando o mesmo ponto inicial for utilizado [2].

Atualmente, o Steepest Descent é um método utilizado com frequência para a resolução de um novo problema, assim como na utilização deste como padrão de referência na medição de outras técnicas [6].

Neste algoritmo de otimização de primeira ordem, para que o mínimo da função seja alcançado o passo de minimização é dado na direção oposta a direção do gradiente da função [5]. Este processo iterativo é dado por:

$$
x_{k+1}=x_{k}-\alpha_{k} v f \stackrel{(}{()}
$$

O método Simulated Annealing (SA), também chamado de Recozimento Simulado é uma técnica metaheurística de busca local, originada do processo de recozimento de metais. Nesse processo os metais são aquecidos até uma temperatura acima do seu ponto de fusão e posteriormente são resfriados lentamente de forma a se organizarem em estruturas cristalinas livres de defeito [2] até que o ponto de solidificação seja alcançado, quando o sistema atinge um estado de energia mínima [7].

Em outras palavras, o método de recozimento busca atingir esse baixo nível de energia interna do material. No entanto, baixa temperatura não significa que serão obtidos baixos níveis de energia. Se a temperatura inferior de um metal fundido for obtida através de processo lento de resfriamento, obtém-se um cristal puro com um nível baixo de energia. Porém, caso se tenha resfriamento mais rápido, o cristal obtido terá um nível mais alto de energia (material temperado) o que produzirá propriedades mecânicas diferentes no material [8].

Chama-se de métodos de busca local clássicos, quando a partir da solução atual $\left(f_{1}\right)$ é gerada uma nova solução-tentativa $\left(f_{0}\right)$, e esta por possuir um valor menor substitui a anterior. Na técnica de recozimento simulado, mudanças para soluções de maior valor são admitidas conforme uma determinada probabilidade, Equação 2, evitando assim que o método fique preso em um mínimo local. Esta probabilidade está ligada à temperatura do sistema físico original, e diminui com o passar das iterações [2].

Prob $=\mathrm{e}^{\left(f_{1}-f_{0}\right) / T}$

\section{Metodologia}

O presente trabalho tem como intuito minimizar a função proposta por Orlande et al. [9], cujo seu comportamento não pode ser analisado, sendo esta denominada de função caixa preta. Para tanto, foram atribuídos valores iniciais para as variáveis da função objetivo, que possui quatro parâmetros de entrada, com a finalidade de obter a saída desta função.

Primeiramente, determinou-se que os valores iniciais da função objetivo seriam $P=\left[\begin{array}{llll}0 & 0 & 0 & 0\end{array}\right]$. E em seguida utilizaram-se o método de Steepest Descent que 
baseia-se no gradiente da função cujo movimento é realizado em apenas um sentido e o método de Simulated Annealing, que diferentemente do método anterior pode mover-se em qualquer sentido, saindo de possíveis mínimos locais.

Com base nos fluxogramas apresentados nas Figuras 1 e 2 que descrevem os métodos Steepest Descent e Simulated Annealing, respectivamente, foi possível implementá-los no MatLab.

Para o método Steepest Descent, após a determinação do ponto inicial, calculou-se o gradiente da função e decidiu-se que o sentido a ser percorrido seria contrário

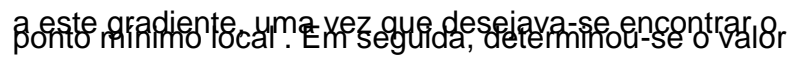
de alfa, tamanho do passo dado em direção ao objetivo, sendo o valor estabelecido de $10^{-3}$, segundo a literatura. Assim, calculou-se um novo valor para o ponto $P$ seguindo a equação (1), e com este novo ponto pode-se calcular o novo valor da função objetivo $\left(S\left(P^{k}\right)\right)$.

Deste modo, se a função objetivo fosse menor do que a tolerância estabelecida de acordo com a literatura, de $10^{-6}$, o ponto seria aceito como valor ótimo. Se a função fosse maior que a tolerância, era necessário fazer algumas verificações: 1- se o número de iterações (kmax), que neste caso foi de 30.000 iterações, fosse alcançado, o programa chegava ao fim mesmo sem encontrar o ponto ótimo, 2- se a função atingisse um valor esperado, o programa chegava ao fim, e 3- se o gradiente da nova função chegasse a um valor esperado, também levaria ao fim do programa. Porém, se nenhum desses critérios fosse atendido, era preciso voltar ao passo de cálculo do gradiente ( $\nabla S\left(P^{k}\right)$ ) utilizando a nova função relacionada ao número da iteração $(k)$ correpondente e repetindo os passos descritos anteriormente.

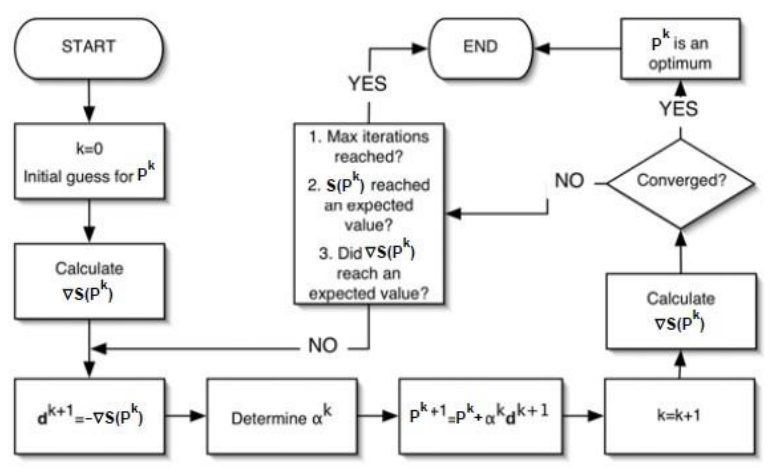

Figura 1: Fluxograma do método Steepest Descent [9]

Para o método Simulated Annealing, após a determinação do ponto inicial, calculou-se o respectivo valor da função neste ponto. Em seguida determinouse a temperatura inicial $(T=700 \mathrm{~K})$, a taxa de redução da temperatura $(R T=0,9)$, o passo de procura $(V=$ 0,03), o número de ciclos ( $N_{s}=100$ ), o número de iterações ( $N_{i t}=75$ ) e um vetor randômico $\left(R_{i}\right)$, entre 1 e 1 . E assim a cada iteração gerou-se um novo valor de $P$, que é calculado conforme a Equação 3 :

$$
P_{i}^{1}=P_{i}^{0}+R_{i} V
$$

Assim, com o novo valor de $P$, encontrou-se o novo valor da função. Se a nova função $\left(U^{1}\right)_{i}$ fosse menor que a anterior (

$\left.U_{i}\right)$ valor de $P$ respectivo seria aceito, e assim a função moveria-se para baixo. Caso a nova função fosse maior que a anterior, esse ponto não seria rejeitado, mas usaria-se um critério probabilístico para decidir se seria aceito ou não, conforme a Equação 2. Ao entrar nesse critério, um número randômico $(r)$, entre 0 e 1, seria gerado, e o valor da probabilidade ( Prob ) seria comparado com o $r$. Assim se Prob $<r$, o ponto seria aceito e a função moveria-se para cima, se Prob $>r$, o valor seria rejeitado.

Esses passos seriam repetidos até que o valor total das iterações fosse alcançado. A cada ciclo o passo de procura $(V)$ é modificado, e a cada iteração a temperatura também é modificada, conforme a Figura 2.

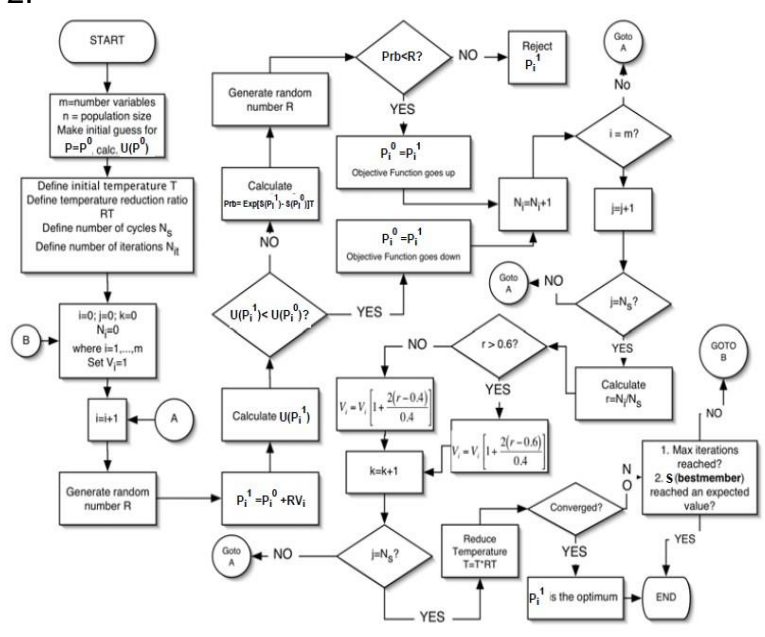

Figura 2: Fluxograma do método de Simulated Annealing[9]

\section{Resultado e Discussão}

Finalizando a simulação de cada método pode-se observar que o método Steepest Descent, obteve o valor ótimo de Snew $=2,2330 \cdot 10^{-5}$, de acordo com os valores iniciais adotados. Porém no método 
Simulated Annealing, não foi possível obter um valor fixo para a função $U$ da caixa preta, pois a cada simulação era gerado um valor diferente, mesmo com os valores iniciais sendo fixos. Isso ocorreu devido à presença de números randômicos que fazem com que os valores se alterem.

A figura 3 descreve o comportamento da função caixa preta de acordo com o método Steepest Descent.

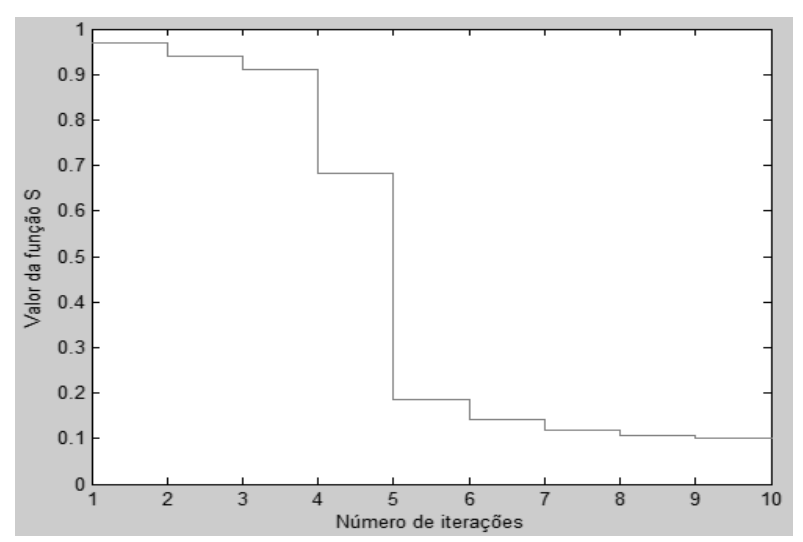

Figura 3: Simulação do método Steepest Descent

Analisando a Figura 3 obtida, é possível notar que por este método sempre será encontrado um valor menor que o anterior para a função, durante as iterações, e essa busca pelo valor mínimo continuou até que fosse atingido um dos critérios de parada já descritos anteriormente. É importante ressaltar que o número de iterações desse processo foi de 24.829, podendo-se concluir assim que o limite de iterações não foi atingido uma vez que o valor máximo era de 30.000 iterações, portanto este não foi o critério de parada do programa em análise. O tempo de simulação desse método foi de aproximadamente 3,172363 segundos.

Uma das soluções gerada no método Simulated Annealing, é mostrada na Figura 4. Pode-se observar que a função não apenas decresce, mas possui pontos onde a mesma assume valores maiores que a anterior para sair dos possíveis mínimos locais. Nessa solução, temos que o valor mínimo encontrado ao final do programa, foi de $U=0,9870$. Utilizando uma função do Matlab, chamada MIN, que para um conjunto de vetores determina o menor elemento existente, e dentro de matrizes é um vetor linha que contém o menor elemento de cada coluna, determinou-se que o menor valor da função caixa preta foi de $U=0,9309$, o que também pode ser percebido na Figura 4. Porém o método saiu desse possível mínimo global à procura de um melhor valor mínimo da função existente, o que permite que mínimos locais sejam rejeitados e a busca por um mínimo global continue. Entretanto, o método Simulated Annealing não retorna a um valor já encontrado anteriormente, então o emprego da função
MIN se faz necessário, uma vez que o mínimo global já possa ter sido encontrado.

Com o número de ciclos, o número de iterações e o número de variáveis da função, preestabelecidos, o método SA realiza 30.001 iterações. O valor da temperatura ao final de cada simulação sempre será o mesmo, $T=0,2590 \mathrm{~K}$, uma vez que a taxa de redução da temperatura e o número de iterações é o mesmo em cada simulação. Assumiu-se a taxa de redução da temperatura igual a 0,9 , para que o decréscimo da temperatura fosse pequeno, e o passo de procura igual a 0,03 para que a função percorresse pequenas distâncias e não desprezasse algum mínimo. O tempo de simulação desse método foi de aproximadamente 0,713190 segundos.

Adicionou-se ao algoritmo um contador, $\mathrm{Nr}$, que tem a função de registrar os valores rejeitados, quando o critério de probabilidade de um ponto é acionada, e o mesmo não é aceito. Em outras palavras, $N r$ é quantas vezes um valor pior para a função foi rejeitado. Nessa solução, temos $N r=6$, porém sabe-se que à medida que a temperatura ( $T$ ) diminui ou quando que a solução atual é maior que a solução-tentativa, ou seja, $f 1$ - fo aumenta, a probabilidade de aceitar o ponto diminui, assim Nr poderá ser maior.

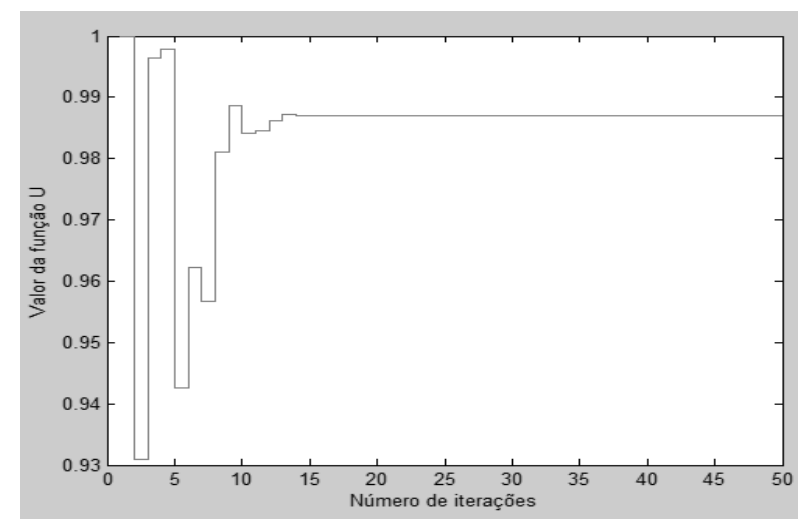

Figura 4: Simulação do método Simulated Annealing

\section{Conclusão}

Através dos dados apresentados, percebe-se que o método Steepest Descent apresentou um menor valor para a função caixa preta quando comparado ao método Simulated Annealing, com os mesmos valores iniciais para a função. Entretanto, esse método não garante que o valor encontrado é um mínimo global, uma vez que a simulação é finalizada quando o valor da função é maior do que seu valor anterior, ou seja, o método encontra o mínimo mais próximo do chute dado inicialmente. Dessa forma, mesmo o método Simulated Annealing não apresentando o melhor valor para a função ao final dessa simulação, este método encontra mínimos e consegue sair deles para continuar a procura pelo mínimo global. Assim, para que haja uma 
maior probabilidade do método SA encontrar o mínimo global da função é necessário o aumento do domínio de procura para que se consiga varrer um espaço maior, porém o gasto computacional para tal fato é elevado. Vale destacar, que com o mesmo número de iterações, o método SA possui um gasto computacional menor que o método Steepest Descent, isso porque o tempo gasto na sua simulação foi menor

\section{Referências}

[1] STELLE, C.; MAIA FILHO, G. R. e SANTI, G. DE C., 2015. Aplicação da Técnica Recozimento Simulado (Simulated Annealing) para Otimização de Reservatórios de pequenas centrais hidrelétricas em Cascata. Universidade Tecnológica Federal do Paraná. Curitiba.

[2] BISCAIA, E. C., 2014. Otimização de Processos I. Disponível em: http://www2.peq.coppe.ufrj.br. Acesso em 15 de junho de 2016.

[3] ROMERO, R. e MANTOVANI, J. R. S., 2004. Introdução a Metaheurística. In: 3ํㅡㄹ Congresso Temático de dinâmica e controle da SBMAC. Ilha Solteira. Anais...

[4] ROUSE, M., 2008. Black box (black box testing). Diaponível
$<$ http://searchsoftwarequality.techtarget.com>. Acesso em 15 de junho de 2016.

[5] ABUD FILHO, Emilio. Aplicação de métodos varacionais e formulações heurísticas para análise e síntese numérica de transformadores em guia de onda retangurales. 2010. Dissertação de Mestrado. PUC-Rio, Rio de Janeiro.

[6] LUENBERGER, D. G.; YE, Y. Linear and Nonlinear Programming. 4a․ ed. Nova lorque, EUA: Springer Science+Business Media, LLC, 2008.

[7] PEREIRA, G. W., 2014. Aplicação da Técnica de Recozimento Simulado em Problemas de Planejamento Florestal Multiobjetivo. Universidade Federal de Minas Gerais. Belo Horizonte.

[8] SOEIRO, F. J. C.P.; CARVALHO, G. de e SILVA NETO A. J. da, 2000. Estimativa de Propriedades Térmicas de Materias Poliméricos com o Método do Recozimento Simulado. Instituto Politécnico, Universidade Federal do Rio de Janeiro. Nova Friburgo.

[9] ORLANDE, H. R.B.; FUDYM, O.; MAILLET, D. e COTTA, R. M. Thermal Measurements and Inverse Techniques. Boca Raton, Florida. Taylor \& Francis Group, LLC, 2011. 